\title{
Interfacial molecular layering enhances specific heat of nanofluids: evidence from molecular dynamics
}

Article

Accepted Version

Creative Commons: Attribution-Noncommercial-No Derivative Works 4.0

Carrillo-Berdugo, I., Grau-Crespo, R. ORCID:

https://orcid.org/0000-0001-8845-1719, Zorrilla, D. and Navas, J. (2021) Interfacial molecular layering enhances specific heat of nanofluids: evidence from molecular dynamics. Journal of Molecular Liquids, 325. 115217. ISSN 0167-7322 doi: https://doi.org/10.1016/j.molliq.2020.115217 Available at https://centaur.reading.ac.uk/97102/

It is advisable to refer to the publisher's version if you intend to cite from the work. See Guidance on citing.

Published version at: https://doi.org/10.1016/j.molliq.2020.115217

To link to this article DOI: http://dx.doi.org/10.1016/j.molliq.2020.115217

Publisher: Elsevier

All outputs in CentAUR are protected by Intellectual Property Rights law, including copyright law. Copyright and IPR is retained by the creators or other copyright holders. Terms and conditions for use of this material are defined in the End User Agreement. 


\section{CentAUR}

Central Archive at the University of Reading

Reading's research outputs online 
Interfacial molecular layering enhances specific heat of nanofluids: evidence

\title{
from molecular dynamics
}

\author{
Iván Carrillo-Berdugo, ${ }^{\dagger}$ Ricardo Grau-Crespo, ${ }^{\dagger}$ David Zorrilla, ${ }^{\dagger}$ Javier Navas ${ }^{\dagger, *}$ \\ † Departamento de Química Física, Facultad de Ciencias, Universidad de Cádiz, E-11510 Puerto Real (Cádiz), Spain. \\ $¥$ Department of Chemistry, School of Chemistry, Food and Pharmacy, University of Reading, United Kingdom, RG6 \\ $6 \mathrm{AD}$
}

*Corresponding author: javier.navas@uca.es (J. Navas) 


\begin{abstract}
It is well known that the specific heat of a heat transfer fluid can be significantly improved by the addition of nanoparticles to form a so-called nanofluid, but the origin of the effect is not completely understood yet. Here, we use molecular dynamics simulations in a nanofluid consisting of palladium nanoparticles in a commercial heat transfer fluid, to demonstrate that a significant fraction (nearly half) of the specific heat enhancement associated to the presence of the nanoparticles is due to strong chemisorption interaction of the fluid molecules at the nanoparticle surfaces. This insight opens new avenues for the nanofluid research community by providing sufficient understanding on the role of chemical interactions in the specific heat of nanofluids, helping on the discussion of what materials and base fluids to be chosen for the optimisation of the thermophysical properties of nanofluids and promoting the transition from basic research to actual application in energy conversion and thermal management processes.
\end{abstract}

\title{
Keywords
}

Nanofluids; specific heat; solid-liquid interface; adsorption; density functional theory; molecular dynamics 

Abbreviations
HTF heat transfer fluid
BP biphenyl
DPO diphenyl oxide
Pd-AO Pd-containing aromatic oil-based nanofluid
MD molecular dynamics
OPLS-AA Optimised Potential for Liquid Simulations - All Atoms forcefield
PES potential energy surface
DFT density functional theory
VASP Vienna Ab-initio Simulation Package
GGA generalised gradient approximation
PBE-D3 Perdew-Burke-Ernzerhof functional with Grimme's D3 dispersion correction
PAW projector augmented wave pseudopotentials
GULP General Utility Lattice Programme
LAMMPS Large Atomic/Molecular Massively Parallel Simulator
PPPM particle-particle particle-mesh
RDF radial distribution function

\section{Symbols}

$\begin{array}{ll}E & \text { energy } \\ d & \text { interplanar spacing } \\ G(r) & \text { molecular radial distribution function } \\ g_{i j}(r) & i j \text { pair radial distribution function } \\ r & \text { radial distance } \\ \alpha & \text { atomic fraction } \\ c & \text { specific heat } \\ k_{\mathrm{B}} & \text { Boltzmann's constant } \\ T & \text { temperature } \\ c_{\chi}^{\prime} & \text { specific heat contribution from energy partition term } \chi \\ \Delta c_{\chi}^{\prime} & \text { excess specific heat contribution from energy partition term } \chi\end{array}$




\section{Introduction}

The use of nanofluids $[1,2]$ as an advanced generation of heat transfer fluids (HTF) is a cost-saving passive technique for the purpose of optimisation of heat transfer processes in thermal management and energy conversion applications [3]. There are many studies dedicated to the characterisation of the stability and those physical properties of nanofluids that rule the heat transfer process, mainly thermal conductivity, dynamic viscosity, specific heat and density. However, comparative studies are usually inconclusive on the magnitude of potential benefits in heat transfer using nanofluids, which makes benchmarking unattainable, if not impossible. An insufficient understanding of nanofluid systems is ultimately translated in a huge obstacle for the transition from basic research to actual application [4]. The influence of factors such as the chemical nature, concentration, size distribution or morphology of nanoparticles in the disperse phase, which can all be controlled ahead of the formulation, have been widely addressed. By contrast, the structure and behaviour of the solid-liquid interface, which appears once the nanofluid is formulated, receives little attention. If the properties of the interfacial fluid are different to the bulk fluid, considering the large exposed surface of the disperse phase in nanofluids, the existence of a unique solid-liquid interface will significantly contribute to the overall nanofluid properties. The complexity of nanofluid systems is underestimated at this point.

The solid-liquid interface has been proposed to play a key role for the anomalous enhancement of specific heat in some nanofluids, for which experimental data exists [5-9]. Particular emphasis should be put on such anomaly, since the most widely used numerical models for the specific heat of mixtures fail to predict it [10]. These models are usually based on or derived from the effective medium approach, meaning no information on the microscopic structure of the mixture has been considered. More recently, Shin and Banerjee [11] identified the higher amplitude of vibrations between atoms at the nanoparticle surface together with solid-fluid interfacial interactions as the root cause for enhanced specific heat in nanofluids. The interfacial layer concept was further developed by 
Hentschke [12], who attempted to explain non-monotonous trends of specific heat as a function of the load of nanoparticles, with a distinctive maximum at intermediate concentrations. This phenomenological model provides an approximate description of available data and, more importantly, significance to a very unique feature of the nanofluid microscopic structure.

In previous work [9] we characterised the specific heat of nanofluids with Dowtherm ${ }^{\mathrm{TM}} \mathrm{A}$ (the eutectic mixture of biphenyl (BP) and diphenyl oxide (DPO), a HTF for solar thermal industry) as base fluid and Pd nanoplates as a disperse phase. The thermophysical property of interest was characterised as a function of temperature and mass fraction of Pd nanoplates. It was found to increase with respect to the base fluid, with a maximum for specific heat for intermediate mass fractions in all cases. We assessed the feasibility for such interfacial layering to occur on these nanofluids via DFT simulations. The chemisorption of BP and DPO was determined to be thermodynamically favoured, with an activation energy requirement for the adsorption of DPO on Pd (111) and Pd (100) surfaces. Even if the existence of the interfacial molecular layer was proven to exist, no unequivocal direct link between the existence of such layer and the specific heat enhancement observed in of Pd-containing aromatic oil-based (Pd-AO) nanofluids was provided. Such is the scope of the present work. In order to assess the influence of such a layer on the specific heat of Pd-AO nanofluids, different models with physisorption-induced and chemisorption-induced interfaces have been investigated using molecular dynamics (MD). This has allowed to understand the effect of strong nanoparticle-fluid interaction on the specific heat of nanofluids, as a function of chemisorption coverage and temperature.

\section{Methodology}

\subsection{Initial configurations and force field details}

We have performed classical MD simulations [13] to investigate the effect of interfacial molecular layering on the specific heat of Pd-AO nanofluids. The simulated systems are summarised in Figure 1. 
The force field for each model system are described below. For a more detailed view on the force field parametrisation, see Tables S1-S5 in the Supplementary Material.

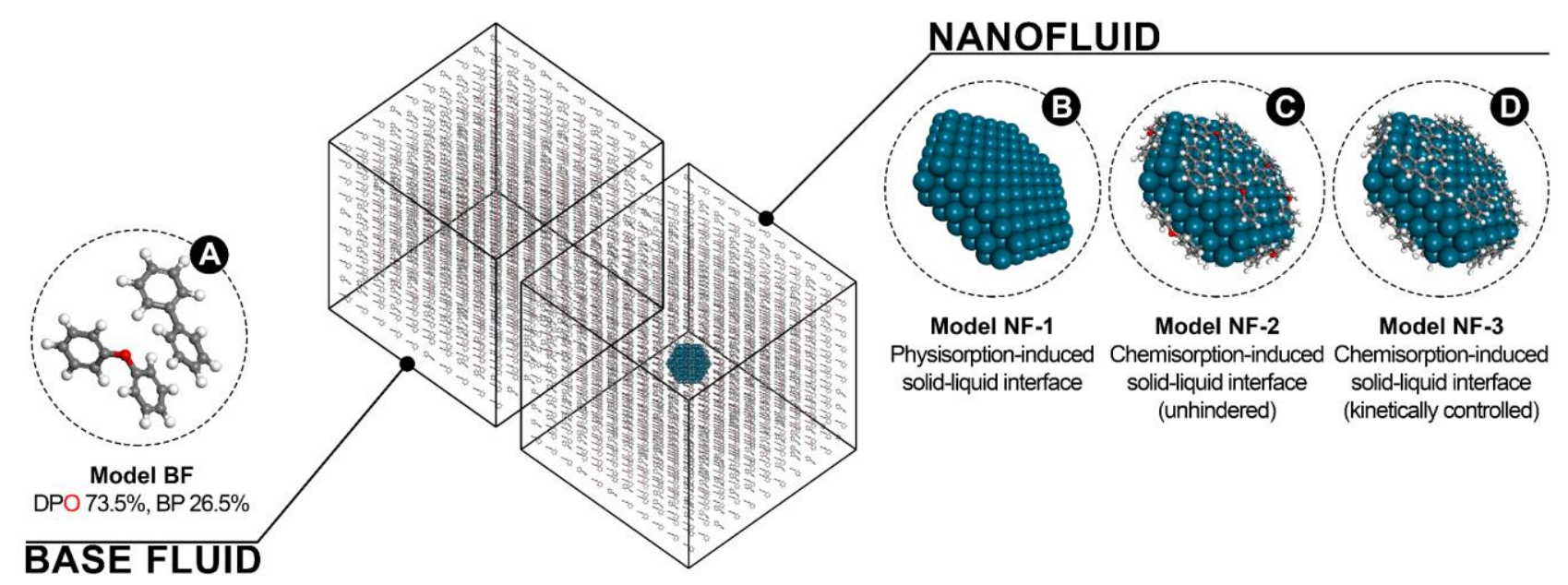

Figure 1. Summary of MD models for $[\mathrm{A}]$ the base fluid (BF) and nanofluids different solid-liquid interfaces induced by [B] physisorption (NF-1), [C] unhindered chemisorption (NF-2) and [D] kinetically controlled chemisorption (NF-3).

The first model system is the base fluid model (BF), shown in Figure 1A, represented by a box of 265 BP molecules and 735 DPO molecules, preserving the eutectic proportion. The Optimised Potential for Liquid Simulation - All Atom (OPLS-AA) force field [14] with parameters from LigParGen [1517] was considered for bonding and non-bonding interactions between atoms in the base fluid. The Lorentz-Berthelot combining rules are used to parametrise the Lennard-Jones potential for pairwise interactions between odd atoms. All atoms are explicitly included (no dummy atoms are used).

The second model is a Pd-AO nanofluid model with a physisorption-induced solid-liquid interface (NF-1), shown in Figure 1B, in which the Pd nanoplate is initially uncovered and surrounded by 1206 base fluid molecules. The Pd nanoplate is represented as an assembly of $241 \mathrm{Pd}$ atoms in ABCstacking and faceted by (111) and (100) surfaces, forming a hexagonal-shaped thin nanostructure 
with $1.4 \mathrm{~nm}$ in edges and $0.5 \mathrm{~nm}$ in thickness. The edge-to-thickness aspect ratio of this nanoplate model is almost equivalent to the aspect ratio of the nanoplates synthesised in our previous work [9]. A Morse potential with parameters from Yokoyama and Ohta [18] is introduced to account for bonding interactions between $\mathrm{Pd}$ atoms in the nanostructure. The pairwise interactions between $\mathrm{Pd}$ atoms [19] and base fluid atoms in this model are described by the Lennard-Jones potential, which is again parametrised using the Lorentz-Berthelot combining rules. No chemical interactions, beyond van der Waals effects represented in the Lennard-Jones potential, are allowed between the nanoparticle and the fluid molecules in this model.

The third and fourth models for this study are those of a Pd-AO nanofluid with a chemisorptioninduced solid-liquid interface, represented as a surface-decorated Pd nanoplate surrounded by 1206 base fluid molecules. In the third model (NF-2), shown in Figure 1C, chemisorption is assumed to occur freely, with no activation energy requirements, hence both BP and DPO are chemisorbed with a fractional coverage determined by the relative proportion of the species in the eutectic mixture. In the fourth model (NF-3), shown in Figure 1D, chemisorption is treated as a kinetically-controlled process, thus one of the molecules is predominantly chemisorbed over the other one. It is known, in fact, that an activation energy requirement in the order of $\sim 0.2 \mathrm{eV}$ exists for the adsorption of DPO and, according to the sticking probabilities at the range of temperatures within $298 \mathrm{~K}$ and $678 \mathrm{~K}$, such a requirement is enough for BP to completely cover the nanoplate surface. A total of 18 chemisorbed molecules are considered in each model, for surface coverage to be maximised and steric repulsions to be kept to a minimum. Also, a maximum of two $\mathrm{C}$ atoms are simultaneously bonded to one $\mathrm{Pd}$ atom. A Morse potential is introduced to account for bonding interactions between Pd atoms on the surface of the nanoplate and $\mathrm{C}$ atoms of chemisorbed base fluid molecules in these models. The parametrisation for such unique interaction is derived from $a b$ initio energy calculations as shown in the following section. 


\subsection{Pd-C interactions at the chemisorption-induced solid-liquid interface}

The quality of the description of the chemisorption phenomenon in a classical MD simulation depends on the accuracy with which the potential energy surface (PES) of the adsorbate-adsorbent interaction is reproduced by the forcefield. Since our goal is to investigate the effect of the chemisorbed layer on the properties of the nanofluid, the accurate parameterisation of this interaction is of crucial importance.

We know from our previous work [9] that the most stable adsorbate-adsorbent configurations are $\mathrm{Bri}_{30}-\mathrm{Bri}_{30}$ for both DPO and BP on the Pd (111) surface and $\mathrm{H}^{\mathrm{bcc}}-\mathrm{H}^{\mathrm{bcc}}$ for both DPO and BP on the Pd (100) surface, respectively. The geometry of these configurations (shown in Figure S1 in the Supplementary Material, together with the meaning of adsorption site nomenclature) was optimised via periodic density functional theory (DFT) simulations. A PES can be generated by scanning the Pd-C bond with single-point energy calculations for different distances from the equilibrium bond length. The optimised geometry of the adsorbed molecules was kept fixed. The Vienna Ab-Initio Simulation Package (VASP) [20-23] was used to perform all periodic-DFT simulations. We used supercells representing surface terminations with a vacuum slab of $15 \AA$, to minimise unphysical interactions with periodic images in the direction perpendicular to the surface. The exchangecorrelation term was calculated within the generalized gradient approximation (GGA) using the dispersion-corrected Perdew-Burke-Ernzerhof (PBE-D3) functional [24, 25]. Brillouin-zone integrations were performed using Monkhorst-Pack grids $[26,27]$ with a k-point density of $0.2 \AA^{-1}$ and a first-order Methfessel-Paxton smearing [28] of $0.2 \mathrm{eV}$. Interactions between valence electrons and atomic cores were described using projector augmented wave (PAW) potentials. The kinetic energy cut-off of the plane-wave basis set expansion was set at $400 \mathrm{eV}$, which is the default for the employed PAW potentials [29, 30]. The parameters for a Morse potential are derived from these PES using the fitting feature available in the General Utility Lattice Program (GULP) [31-35], which considers the Newton-Raphson functional minimisation approach for such a purpose. For a more 
representative interatomic potential for the $\mathrm{Pd}-\mathrm{C}$ interaction, potential parameters are successively averaged and refined. More details on the derivation of this interatomic potential together with an analysis of the distribution of energy residuals is presented in Tables S6-S8 in the Supplementary Material.

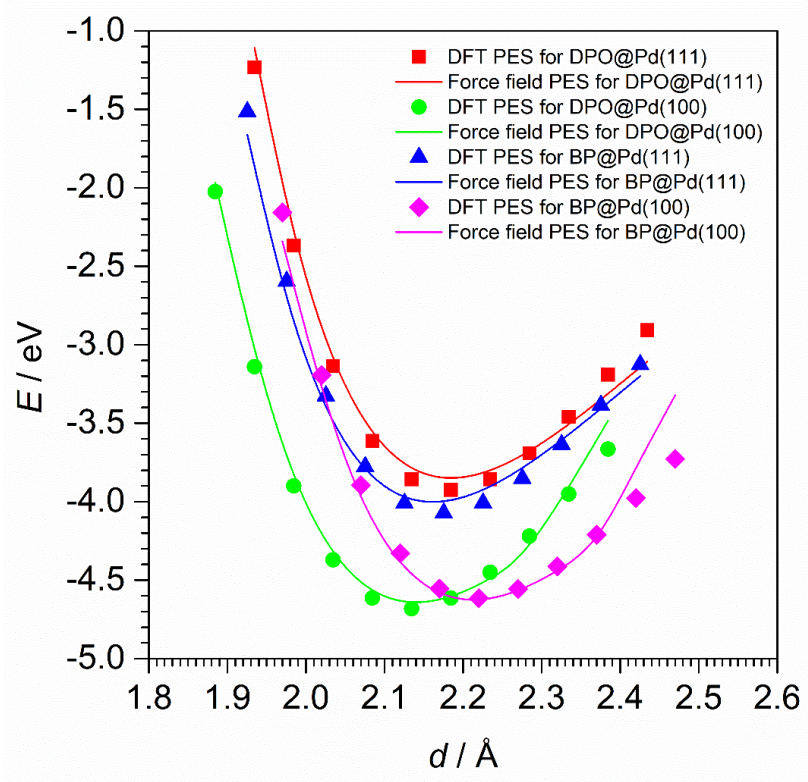

Figure 2. DFT PES (scattered dots) for the interaction of the fluid molecules palladium surfaces, in comparison with the generated force field PES (solid lines). $d$ is the interplanar spacing between the metallic surface and the planes defined by phenyl rings in the molecules.

Thus, a total of four DFT PES have been generated, for the chemisorption of each molecule on each surface, and a Morse potential function for $\mathrm{Pd}-\mathrm{C}$ interaction has been derived from them. As it is shown in Figure 2 (also in Figure S2 in the Supplementary Material), the force field describes the PES with excellent agreement, to the extent of being able to reproduce and distinguish fine details such the adsorption energy or the distance of the chemisorbed molecule from the surface in each case. This Morse potential will keep molecules permanently attached to the surface during the dynamics 
of chemisorption-induced models. This is justified considering the stability of the adsorbateadsorbent complexes has been proven in our previous work on this system [9]. Adsorption energies are in the order of $\sim 4.0 \mathrm{eV}$ and the energetics of thermal effects are in the order of $k_{\mathrm{B}} T$ (i.e. $41 \mathrm{meV}$ at $475 \mathrm{~K}$ ) thus, in the range of operating temperatures, BP or DPO molecules will not leave the solidliquid interface once adsorbed.

\subsection{Molecular Dynamics simulations setup}

The Large-scale Atomic/Molecular Massively Parallel Simulator (LAMMPS) $[36,37]$ is the classical MD code of choice for the comprehensive study on the effect of the interfacial molecular layering on the specific heat of Pd-AO nanofluids. Each of the above explained initial configurations have been created with Moltemplate [38, 39], a text-based molecular builder for LAMMPS. Periodic boundary conditions are imposed in the system. The Verlet integration scheme [40] is used with a timestep of 1 fs. Velocities are rescaled with the Nosé-Hoover thermostat and barostat algorithms [41-44]. Lennard-Jones pairwise interactions are limited to a cut-off distance of $10 \AA$. The particle-particle particle-mesh (PPPM) summation method [45] was applied to compute long-range electrostatics. With this general set-up, the MD simulation procedure involves (i) a 10 ps run in NVE for relaxation, (ii) a 0.5 ns run in NVT for thermalisation, (iii) a 1 ns run in NPT for equilibration and (iv) a 5 ns run in NVT for the computation of density, specific heat, energies and pair radial distribution functions (RDF). Thermodynamic outputs are dumped every 1 ps and pair RDF every 50 ps.

Different series of MD simulations will be carried out with this scheme. The simulation conditions will be first validated by comparing computed results on density and specific heat for the base fluid at 323/373/423/473 $\mathrm{K}$ and $1 \mathrm{~atm}$ with available experimental data from the supplier. The base fluid model and simulation conditions were validated as both density and specific heat were found to be in the same order of magnitude than experimental observations and exhibited the same behaviour versus changes in the state variable temperature (data for validation is presented in Figure S3 of the Supplementary Material). Then the comparison of Pd-AO nanofluid models with different interfacial 
molecular layering considerations is approached by computing the dynamics of each model system at $473 \mathrm{~K}$ and $1 \mathrm{~atm}$. The dynamics of the most representative nanofluid model will be again computed at $473 \mathrm{~K}$ and $1 \mathrm{~atm}$ but with different numbers of chemisorbed molecules, for an analysis on the dependence of specific heat of this nanofluid as a function of the number of occupied adsorption sites. Alternatively, the dynamics of the most representative nanofluid model system will also be computed $323 / 373 / 423 / 473 \mathrm{~K}$ and 1 atm for a comparative analysis of the specific heat of this nanofluid and the base fluid as a function of temperature.

\section{Results and discussion}

\subsection{Molecular architecture from radial distribution functions}

The purpose of this paper is to approach the effect of the interfacial molecular layering on the specific heat of Pd-AO nanofluids by multiple-model comparison at the MD level of theory. The dynamics of three different models with different representations of the solid-liquid interface (see Figure 1) have been computed: a physisorption-induced interface (NF-1), an unhindered chemisorption-induced interface (NF-2) and a kinetically-controlled chemisorption-induced interface (NF-3). The molecular architectures at equilibrium are discussed in this section on the basis of the RDFs. We are particularly interested on the molecular RDFs, $G(r)$,

$$
G(r)=\sum_{j} \alpha_{j} g_{i j}(r)
$$

which is a sum of the individual pair RDFs of atom $j$ (any atom in base fluid molecules) with respect to atom $i$ (any atom in the nanoplate), $g_{i j}(r)$, weighted by the atomic fraction of atom $j, \alpha_{j}$. The molecular RDFs are more suitable than individual pair RDFs for the purpose of this paper, which is to describe the molecular architecture formed by base fluid molecules around Pd nanoplates and its relation with the specific heat of Pd-AO nanofluids. $G(r)$ for all nanofluid models are included in 
Figure $3 \mathrm{~A}$ for discussion and $g(r)$ for $\mathrm{Pd}-\mathrm{C}$ and $\mathrm{Pd}-\mathrm{O}$ interactions are included in Figure $\mathrm{S} 4$ for verification purposes.

On the one hand, the molecular RDF for model NF-1, for which a physisorption-induced solid-liquid interface was expected, reveals three peaks, at $3.45 \AA$, $5.95 \AA$ and $7.95 \AA$, whose locally higher number density. This suggests the solely presence of the Pd nanoplate, mediated by the Van der Waals forces (whose effects are included in the model in the form of a classical Lennard-Jones potential), induces some ordering on the surrounding molecules, creating an interface with two solvation layers of base fluid molecules. On the other hand, the molecular RDFs for models NF-2 and NF-3, for which a chemisorption-induced solid-liquid interface was expected, show analogous features in both cases, but quite different compared to model NF-1. First, a very sharp peak with the highest number density appears at $2.15 \AA$. The position of the first peak matches the Pd-C equilibrium bond length of the parametrised Morse potential, so this first peak can be directly assigned to bonding Pd-C interactions. Then, a sequence of peaks follows up to the cut-off distance. As shown in Figure 3B, in which the molecular RDFs of NF-2 and NF-3 models are split into the contributions from adsorbed and nonadsorbed molecules, this sequence is only due to adsorbed molecules, which means it cannot be interpreted as multiple solvation layers. Up to $6.0 \AA$, the position of the peaks in this sequence matches the distances between a given surface $\mathrm{Pd}$ atom of a certain adsorption site and $\mathrm{C}$ atoms of the chemisorbed molecule on that site, but not directly bonded to it (as shown in Figure 3C). Any peak further than that can be assigned to the distribution of $\mathrm{C}$ atoms on a different adsorption site with respect to the given surface $\mathrm{Pd}$ atom or to the distribution of $\mathrm{C}$ atoms with respect to a given non-surface Pd atom. The presence of the very sharp first peak and the following sequence of peaks in the molecular RDFs implies that the chemisorbed layer of base fluid molecules in the solid-liquid is preserved during the computed trajectory, which validates the parametrised Morse potential for its purpose. Alternatively, it is reflected in Figure 3B that the presence of this chemisorbed layer imposes a shielding effect on the interaction between the nanoplate and the non-adsorbed surrounding fluid, 
as only one peak appears at $3.65 \AA$ (the second one has vanished, almost completely), further and lower in number density compared to the first peak in the molecular RDF of NF-1 model.
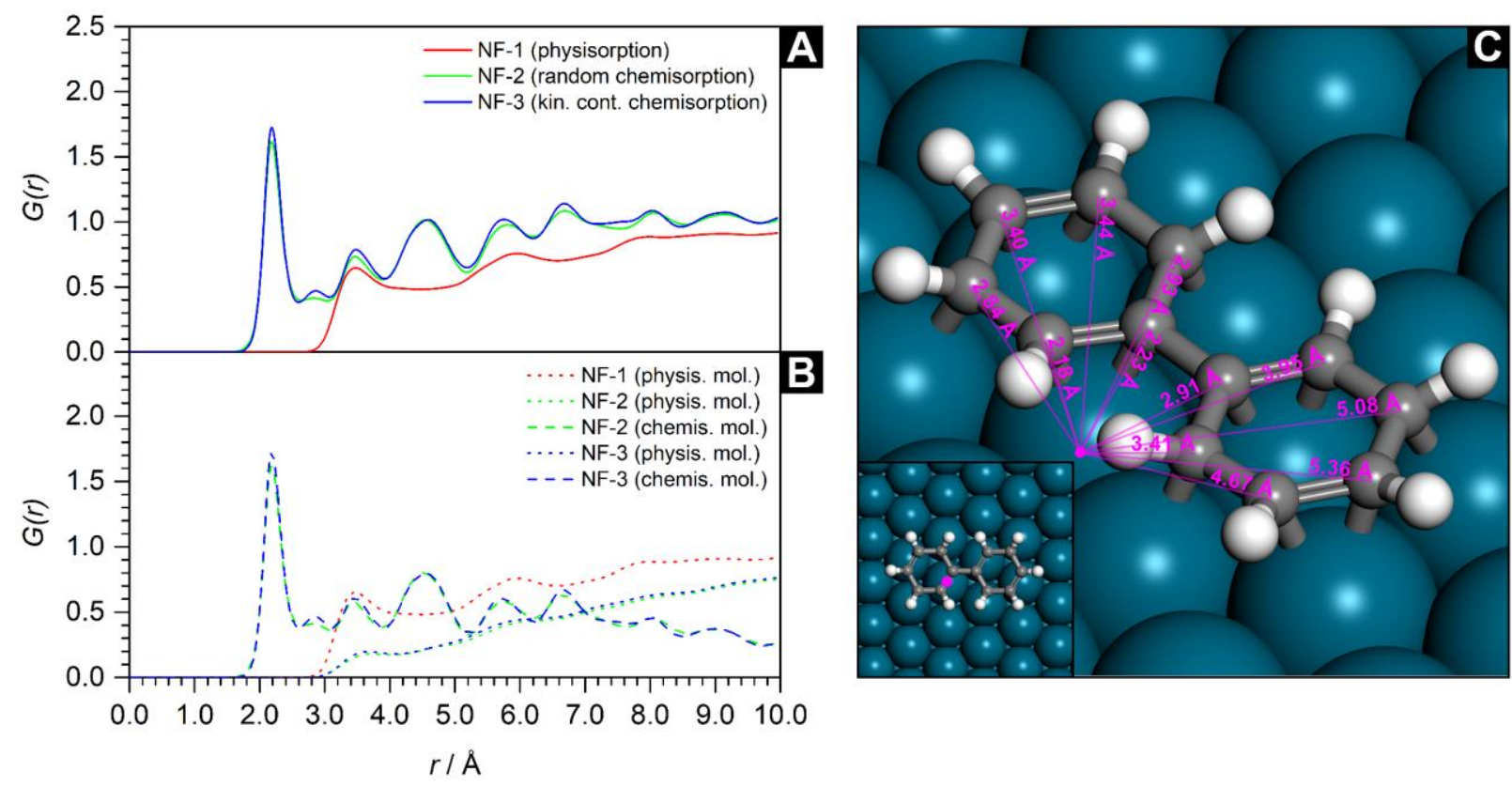

Figure 3. [A] Molecular RDFs for all MD models. [B] Detail of the contributions from physisorbed and chemisorbed species to the molecular RDFs. [C] Example of the distribution of $\mathrm{C}$ atoms with respect to a given Pd atom (distances in this scheme may not exactly match the peaks in the molecular RDF, as this is a snapshot from a particular step in the trajectory of the system).

The main conclusion to be drawn from the molecular RDFs is that molecular layering occurs at the solid-liquid interface of nanofluids, with notable difference if it is considered to be induced by physisorption or chemisorption phenomena, but still occurs. Two interfacial molecular layers appear in both cases, but in the chemisorbed-induced interface the first layer is closer, by $1.3 \AA$, than in the physisorbed one. The consequences of the closer distribution of base fluid molecules around the nanoplate are immediately reflected in density. Computed density values in equilibrium at $473 \mathrm{~K}$ are 
$0.87 \pm 0.01 \mathrm{~g} \cdot \mathrm{cm}^{-3}$ for model BF, $0.97 \pm 0.01 \mathrm{~g} \cdot \mathrm{cm}^{-3}$ for model $\mathrm{NF}-1$, and $0.98 \pm 0.01 \mathrm{~g} \cdot \mathrm{cm}^{-3}$ for both model NF-2 and NF-3. The increase in this physical property with respect to the base fluid is $12.5 \%$ for the model NF-1 and $13.0 \%$ for models NF-2 and NF-3. Although the standard deviations limit this discussion, it is remarkable that the densities of model NF-2 and NF-3 (chemisorption) are slightly higher than that of model NF-1 (physisorption). This is because chemisorption induces a negative excess volume effect for adsorbed molecules and density is, therefore, increased. This is directly related to the more confined situation of base fluid molecules in the chemisorption-induced solid-liquid interface, due to the strength of the Pd-C interaction imposed by the Morse potential in comparison to the Lennard-Jones potential.

\subsection{The impact of interfacial molecular layering on the specific heat of nanofluids}

The impact of this interfacial molecular layering on specific heat is now assessed. Specific heat, $c$, can be determined from energy fluctuations in the canonical ensemble (NVT), as denoted by the following fundamental relation from statistical mechanics [13]

$$
c=\frac{\left\langle E_{T}^{2}\right\rangle-\left\langle E_{T}\right\rangle^{2}}{k_{\mathrm{B}} T^{2}}=\frac{\operatorname{Var}\left(E_{T}\right)}{k_{\mathrm{B}} T^{2}}
$$

in which $\operatorname{Var}\left(E_{T}\right)$ is the total energy variance, $k_{B}$ is the Boltzmann's constant and $T$ is the average temperature. Do note the specific heat is intentionally denoted as $c$, with no distinction for isobaric or isochoric specific heats, as the base fluid model and the nanofluid models are treated as incompressible systems. The values of specific heat computed for the base fluid model and each PdAO nanofluid model are included in Figure 4A. In the most basic comparison with respect to the model BF, it can be appreciated that the solely presence of the Pd nanoplate in model NF-1 increases the specific heat by $4.1 \%$, whilst the presence of the same Pd nanoplate with a chemisorbed molecular layer on its surface in models NF-2 and NF-3 increases it by $7.2 \%$ and $6.6 \%$. 
That result already supports the former statements from different authors on the effect of the strong interfacial molecular layering phenomenon on the specific heat of nanofluids and provides a semiquantitative description on how positive is that effect, but the discussion can be taken one step further. Equation 2 establishes a clear relation between the macroscopic observable specific heat and the microscopic quantity internal energy. Our proposal is that such a relation can also be exploited for the quantification of heat storage arising from fluctuations in bond, angle, dihedron, pairwise (Van der Waals and Coulomb) and kinetic terms. This can be achieved by partitioning energy into these terms (which are easily computed with the thermo command in LAMMPS) and calculating their contribution to specific heat, $c_{\chi}^{\prime}$, from the energy variances and covariances associated to each of them. A detailed explanation on this partition of energy and the definition of these specific heat contributions is given in Section V of the Supplementary Material. The excess specific heats by term, $\Delta c_{\chi}^{\prime}=c_{\chi, n f}^{\prime}-c_{\chi, b f}^{\prime}$, are conveniently plotted in Figure 4B, for these contributions to the total specific heat of each nanofluid model to be compared.

The bond, angle and dihedron terms represent the energy stored in force fields due to 1-2, 1-3 and 14 bonding interactions in the system, respectively. With respect to the base fluid, there is a small contribution from the bond term in the model NF-1, arising from $\mathrm{Pd}-\mathrm{Pd}$ bonding interactions in the nanoplate, particularly from surface atoms, as they have a lower coordination numbers and are allowed to vibrate with higher amplitudes. The bond term contribution to specific heat in models NF2 and NF-3 is notably larger than in NF-1, as consequence of the energy fluctuations arising not only from $\mathrm{Pd}-\mathrm{Pd}$ but also from $\mathrm{Pd}-\mathrm{C}$ interactions at the solid-liquid interface. The higher variability in NF-3 may arise from $\mathrm{C}-\mathrm{C}$ tension between phenyl rings in $\mathrm{BP}$, as we verified in DFT calculations that this distance is slightly stretched in order to the symmetry of rings to fit that of the surface [9]. Given no angles nor dihedra interactions were defined in the nanoplate structure, the existence of an associate angle and dihedron term contributing to specific heat nanofluid models can only be associated to base fluid molecules. The negative excess specific heat from angle term is due to lower energy variability in 1-3 bonding interactions in the nanofluids than in the base fluid, which can be 
consequence of a more rigid intra-annular geometry for phenyl rings in the surrounding (either physisorbed or chemisorbed) base fluid molecules. This negative contribution is particularly accused in NF-2, in which the $\mathrm{C}-\mathrm{O}-\mathrm{C}$ angle is also constrained due to chemisorption. Alternatively, the dihedron term causes a positive excess specific heat, due to the energy variability arising from the imposition of a conformational change to adsorbed molecules with respect to their liquid phase conformation. This effect is particularly significant for models NF-2 and NF-3, because chemisorbed molecules suffer huge conformational changes implying distortions of $\mathrm{C}-\mathrm{O}-\mathrm{C}=\mathrm{C}$ dihedron in $\mathrm{DPO}$ and $\mathrm{C}=\mathrm{C}-\mathrm{C}=\mathrm{C}$ dihedron in $\mathrm{BP}$, which are susceptible of increased energy variability.

The pairwise term accounts for the energy stored force fields due to van der Waals and Coulomb interactions between all atoms in the system. This term introduces a positive excess specific heat, as the nanoplate (with either a physisorption-induced or a chemisorption-induced interface) is a source for increased energy variability as it stablishes long-range interactions with the surrounding medium. This effect is present in all nanofluid models, but it is larger in the case of model NF-2 and smaller in the case of model NF-3. These models were created under the assumptions of unhindered and kinetically controlled chemisorption to occur, so that both DPO and BP or only BP molecules are chemisorbed to the surface. The fact that the excess specific heat due to pairwise interactions in model NF-3 is much smaller means energy variability from these interactions is reduced and it can be ultimately interpreted as the BP-covered nanoplate to be strongly bounded to the surrounding molecules of base fluid in a colloidal local molecular network that is less sensitive to change as the MD runs. In other words, the colloidal local molecular network in model NF-3 seems to exhibit a higher stiffness. This is also reflected in the kinetic term, which is again smaller for model NF-3. This kinetic term accounts for the variability in the kinetic energy associated to the different atomic modes of motion in the system (although the average kinetic energy of the system is constant, because temperature is). The kinetic term is responsible for the largest excess positive heat contribution. 

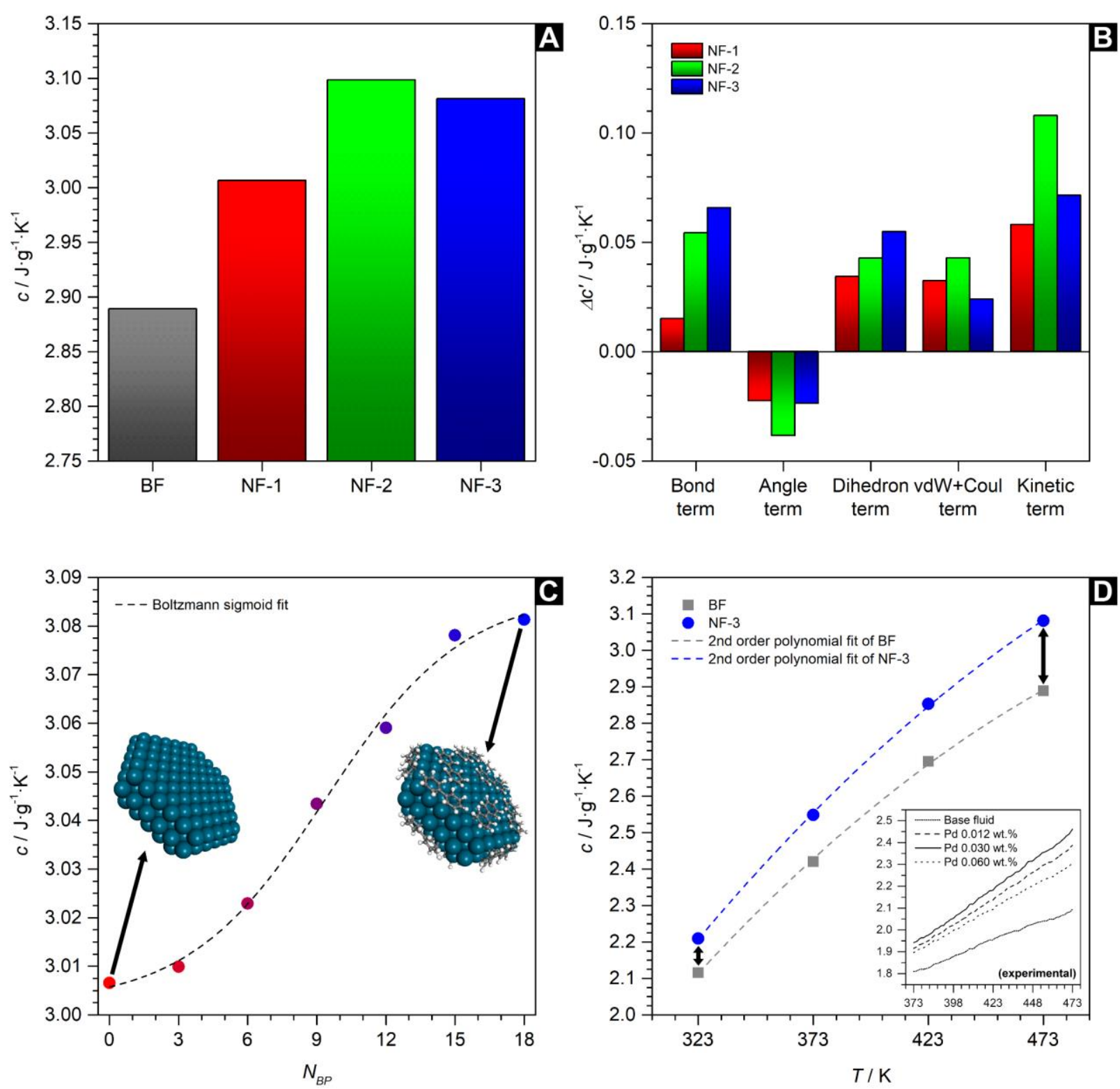

Figure 4. [A] Specific heat values for all models at $473 \mathrm{~K}$. [B] Excess specific heat from each energy term for nanofluid models, with the base fluid as a zero-reference point, at $473 \mathrm{~K}$. [C] Specific heat of model NF-3, as a function of the number of chemisorbed BP molecules. [D] Specific heat of the models BF and NF-3 as a function of temperature (experimental data from reference [9] is included in the inset for comparison purposes). 
Overall, MD simulations revealed significant increases in the specific heat of Pd-AO nanofluid models and the analysis of energy variances and covariances allowed us to identify three sources for energy storage with respect to the base fluid (see Figure 5), explaining those enhancements: surface storage (a low-tier contribution, due to bond interactions between surface $\mathrm{Pd}$ atoms in the nanoplate, vibrating with higher amplitudes), interfacial layer storage (a mid-tier contribution, due to bond interactions between surface Pd atoms and $\mathrm{C}$ atoms in chemisorbed base fluid molecules and also dihedron interactions in conformationally restricted base fluid molecules) and mesolayer storage (high-tier contribution due to pairwise interactions between atoms in the nanoplate, either uncovered or decorated, with atoms in the surrounding base fluid molecules, and also due to the consequent colloidal molecular network stiffness affecting local kinetic energy redistribution).

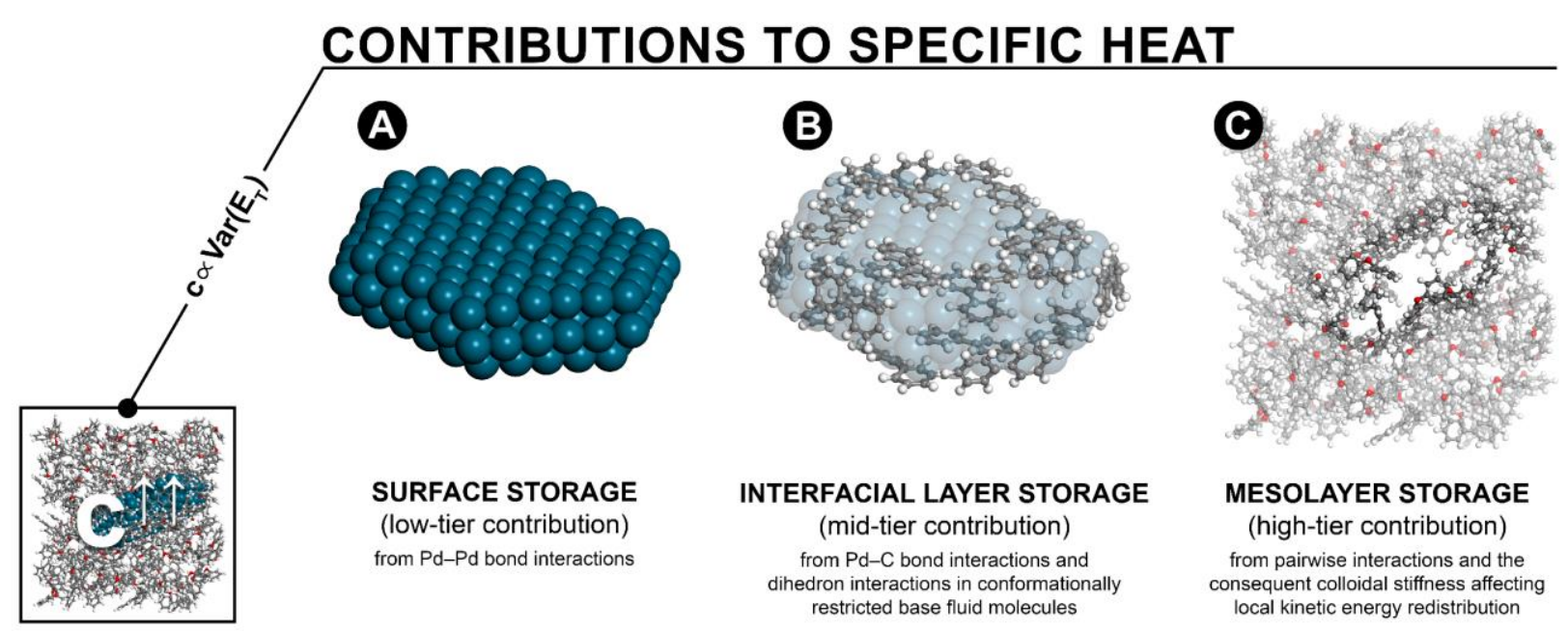

Figure 5. Summary of the contributions to specific heat in nanofluids described in this paper: [A] surface storage, $[\mathrm{B}]$ interfacial layer storage and $[\mathrm{C}]$ mesolayer storage. All structures presented in this figure are part of the same snapshot from MD trajectory. 


\subsection{The response of the specific heat of nanofluids to changes in the number of chemisorbed}

molecules and temperature

We have determined from MD simulations the existence a complex molecular architecture in the solid-liquid interface of nanofluids and how it influences on their specific heat enhancement, all of it supported by mutual feedback with experimental evidence from a previous work [9]. We now focus on the effect of the number of adsorbed molecules on specific heat enhancement. In contrast to the above-presented results, in which we assessed the significance on 'how' molecules are adsorbed, we now focus on the significance of 'how many' molecules are absorbed. For that, we have performed a series of MD simulations using model NF-3, but with different number of BP molecules chemisorbed on the surface of the Pd nanoplate. Given NF-2 and NF-3 are found to have very similar specific heats, the choice of model NF-3 over model NF-2 for these tests is encouraged by the fact that it is the model that considers both the thermodynamics and kinetics of the adsorption processes of DPO and BP on Pd (111) and Pd (100), computed by periodic-DFT simulations.

The computed values for specific heat in MD, as a function of the number of adsorbed BP molecules, are presented in Figure 4C. The plot is bounded between 0 and 18, which is the number of BP molecules in the chemisorbed interfacial layer that saturates the surface of the nanoplate (surface is saturated but not all existing adsorption sites are occupied, because each BP covers two sites but also exerts an excluded volume effect and forbids adjacent sites to be occupied). Specific heat increases for increasing numbers of chemisorbed BP molecules following a sigmoid trend until surface saturation. Each molecule is simultaneously bound to the surface by twelve bonding interactions (in the form of a Morse potential) directly contributing to energy fluctuations and the enhancement of specific heat. This fact strengths the direct link between the existence of a chemisorbed molecular layer and the specific heat enhancement in nanofluids via solid-liquid interfacial storage.

Another significant observation from our previous work [9] are the divergent trends in the specific heat of Pd-AO nanofluid with respect to its base fluid as temperature increases. In other words, the 
enhancement in specific heat due to the addition of Pd nanoplates into the base fluid was observed not to be constant with temperature. This encouraged its study for this paper. Figure 4D gathers the specific heat values derived from MD simulations for the nanofluid model NF-3 (full coverage) and the base fluid model, at $323 \mathrm{~K}, 373 \mathrm{~K}, 423 \mathrm{~K}$ and $473 \mathrm{~K}$. The divergent trends with temperature are well reproduced, as it is evident by comparison with experimental data presented in the inset. The relative enhancements in specific heat (with respect to the base fluid model) are $4.4 \%$ at $323 \mathrm{~K}, 5.3 \%$ at $373 \mathrm{~K}, 5.8 \%$ at $423 \mathrm{~K}$ and $6.6 \%$ at $473 \mathrm{~K}$. In general, higher temperatures make barriers in the energy landscape of the system effectively low and easier to sample during MD simulations, causing an increase in energy fluctuations directly reflected in specific heat. This relation of causality provides a straightforward explanation of the fact that specific heat increases with temperature and it may also explain the divergence in trends with temperature in nanofluids if we pay attention, once again and for the last time, to the interface: the assembled solid-liquid interface structure in nanofluids is much more complex than the structure of the liquid phase, built from very unique interactions shaping a completely new energy landscape which can be expected to be highly disperse in terms of barriers and, thus, more sensitive to changes in temperature. The response to those changes is the energy fluctuations, from which specific heat is derived.

\section{Conclusions}

The interfacial molecular layering concept as a root cause for the anomalous enhancement of specific heat in some nanofluids was postulated and widely accepted among researchers on the field, but under suspect heretofore. Its existence was proved via periodic-DFT simulations of the adsorption energies for the complexes of biphenyl and diphenyl oxide on Pd (111) and (100) surfaces, all of it in a recently published paper by our group. Here we report on its significance of the interfacial molecular layering for the overall nanofluid specific heat, by thoroughly assessing how and how much its existence contributes to this property via MD simulations of different nanofluid models. We have investigated the impact of chemical interactions at solid-liquid interfaces for specific heat by comparing models 
with physisorption-induced and chemisorption-induced interfaces. We concluded that the specific heat of nanofluids, with respect to the base fluid, is enhanced by $4 \%$ in presence of the undecorated nanoplate and by $7 \%$ if the first interfacial molecular layer is chemically bonded to the surface of the nanoplate via $\mathrm{Pd}-\mathrm{C}$ bonds, whose energetics are represented by a Morse potential. The enhancement induced by the interfacial molecular layer was thoroughly investigated by taking advantage of the partition of energy and the definition of excess specific heats from the different partitions. This allowed us to propose a molecular interpretation for the specific heat enhancement in nanofluids in which three sources for energy storage are recognised: surface, interfacial layer and mesolayer storage. We verified that such enhancement is directly proportional to the number of chemisorbed molecules and also magnified for increasing temperatures. Our finding suggests that engineering the interaction between the nanoparticle surfaces and the liquid to promote chemisorption interactions is a promising avenue to further improve the thermophysical properties of nanofluids, in applications such as heat transfer fluids for concentrated solar power, where a large specific heat is desired. It also puts the solid-liquid interface in the spotlight for the research of nanofluids, by demonstrating that the nature of the interactions at the interface has an important effect on the overall nanofluid properties. Although our work illustrates this effect for one particular nanofluid, similar behaviour can be expected for other metal-containing nanofluids where the organic molecules can strongly interact with the metal surfaces, as this is not a behaviour specific to palladium. Our work shows that the magnitude of specific heat enhancement can be dependent on the mode of adsorption (physisorption, non-dissociative chemisorption or even dissociative chemisorption). Clearly, further investigation is needed to establish this phenomenon as a more general one, and as a possible avenue to improve the thermal properties of nanofluids. 


\section{Conflicts of Interest}

The authors declare no competing financial interests.

\section{Acknowledgments}

I.C.-B. acknowledges FPU16/02425 studentship from Ministerio de Universidades del Gobierno de España. We also acknowledge Ministerio de Ciencia e Innovación del Gobierno de España for funding under Grant No. RTI2018-096393-B-I00 and for financial support related to measurements of thermal properties, which were carried out using devices acquired under Grant No. UNCA15-CE2945. This work made use of ARCHER, the UK'S national high-performance computing service, via R.G.-C.'s membership in the HPC Materials Chemistry Consortium, which is funded by EPSRC (EP/R029431). 


\section{References}

[1] H. Masuda, A. Ebata, K. Teramae, N. Hishinuma, Alteration of Thermal Conductivity and Viscosity of Liquid by Dispersing Ultra-fine Particles (Dispersion of $\gamma$-Al2O3, SiO2, and TiO2 Ultrafine Particles), Netsu Bussei 7 (1993) 227-233. http://dx.doi.org/10.2963/jjtp.7.227.

[2] S.U.S. Choi, J.A. Eastman, Enhancing Thermal Conductivity of Fluids with Nanoparticles, ASME IMECE, 1995.

[3] S. Rashidi, M. Eskandarian, O. Mahian, S. Poncet, Combination of nanofluid and inserts for heat transfer enhancement, J. Therm. Anal. Calorim. 135 (2018) 437-460. https://doi.org/10.1007/s10973018-7070-9.

[4] E.V. Timofeeva, W. Yu, D.M. France, D. Singh, J.L. Routbort, Nanofluids for Heat Transfer: An Engineering Approach, Nanoscale Res. Lett. 6 (2011) 182 1-7. https://doi.org/10.1186/1556-276X6-182.

[5] M. Lasfargues, A. Bell, Y. Ding, In situ Production of Titanium Dioxide Nanoparticles in Molten Salt Phase for Thermal Energy Storage and Heat-transfer Fluid Applications, J. Nanopart. Res. 18 (2016) 150 1-11. https://doi.org/10.1007/s11051-016-3460-8.

[6] G. Qiao, M. Lasfargues, A. Alexiadis, Y. Ding, Simulation and Experimental Study of the Specific Heat Capacity of Molten Salt Based Nanofluids, Appl. Therm. Eng. 111 (2017) 1517-1522. https://doi.org/10.1016/j.applthermaleng.2016.07.159.

[7] R. Gómez-Villarejo, E.I. Martín, J. Navas, A. Sánchez-Coronilla, T. Aguilar, J.J. Gallardo, R. Alcántara, D. De los Santos, I. Carrillo-Berdugo, C. Fernández-Lorenzo, Ag-based Nanofluidic System to Enhance Heat Transfer Fluids for Concentrating Solar Power: Nano-level Insights, Appl. Energy 194 (2017) 19-29. https://doi.org/10.1016/j.apenergy.2017.03.003.

[8] Y. Hu, Y. He, Z. Zhang, D. Wen, Enhanced Heat Capacity of Binary Nitrate Eutectic Salt-Silica Nanofluid for Solar Energy Storage, Sol. Energ. Mat. Sol. C. 192 (2019) 94-102. https://doi.org/10.1016/j.solmat.2018.12.019. 
[9] I. Carrillo-Berdugo, S. Midgley, D. Zorrilla, R. Grau-Crespo, J. Navas, Understanding the Specific Heat Enhancement in Metal-containing Nanofluids for Thermal Energy Storage: Experimental and Ab-initio evidence for a Strong Interfacial Layering Effect, ACS Appl. Energy Mater. 3 (2020) 92469256. https://doi.org/10.1021/acsaem.0c01556.

[10] L.-P. Zhou, B.-X. Wang, X.-F. Peng, X.-Z. Du, Y.-P. Yang, On the Specific Heat Capacity of CuO Nanofluid, Adv. Mech. Eng. 2 (2015) 1-4. https://doi.org/10.1155/2010/172085.

[11] D. Shin, D. Banerjee, Enhancement of Specific Heat Capacity of High-temperature Silicananofluids Synthesized in Alkali Chloride Salt Eutectics for Solar Thermal-energy Storage Applications, Int. J. Heat Mass Transfer $54 \quad$ (2011) 1064-1070. https://doi.org/10.1016/j.ijheatmasstransfer.2010.11.017.

[12] R. Hentschke, On the Specific Heat Capacity Enhancement in Nanofluids, Nanoscale Res. Lett. 11 (2016) 88 1-11. https://doi.org/10.1186/s11671-015-1188-5.

[13] M.P. Allen, D.J. Tildesley, Computer Simulation of Liquids. 2nd ed. Oxford University Press, Oxford, UK, 2017.

[14] W.L. Jorgensen, D.S. Maxwell, J. Tirado-Rives, Development and Testing of the OPLS AllAtom Force Field on Conformational Energetics and Properties of Organic Liquids, J. Am. Chem. Soc. 118 (1996) 11225-11236. https://doi.org/10.1021/ja9621760.

[15] W.L. Jorgensen, J. Tirado-Rives, Potential Energy Functions for Atomic-level Simulations of Water and Organic and Biomolecular Systems, Proc. Natl. Acad. Sci. U.S.A. 102 (2005) 6665-6670. https://doi.org/10.1073/pnas.0408037102.

[16] L.S. Dodda, J.Z. Vilseck, J. Tirado-Rives, W.L. Jorgensen, 1.14*CM1A-LBCC: Localized Bond-Charge Corrected CM1A Charges for Condensed-Phase Simulations, J. Phys. Chem. B 121 (2017) 3864-3870. https://doi.org/10.1021/acs.jpcb.7b00272.

[17] L.S. Dodda, I. Cabeza de Vaca, J. Tirado-Rives, W.L. Jorgensen, LigParGen Web Server: An Automatic OPLS-AA Parameter Generator for Organic Ligands, Nucleic Acids Res. 45 (2017) 331336. https://doi.org/10.1093/nar/gkx312. 
[18] T. Yokoyama, T. Ohta, Temperature-Dependent EXAFS Study on Supported Silver and Palladium Clusters: Comparison on Their Interatomic Potentials with Those of Bulk Metals, Jpn. J. Appl. Phys. 29 (1990) 2052-2058. https://doi.org/10.1143/JJAP.29.2052.

[19] H. Heinz, L. T-J, R.K. Mishra, F.S. Emami, Thermodynamically Consistent Force Fields for the Assembly of Inorganic, Organic, and Biological Nanostructures: The INTERFACE Force Field, Langmuir 29 (2013) 1754-1765. https://doi.org/10.1021/la3038846.

[20] G. Kresse, D. Vogtenhuber, M. Marsman, M. Kaltak, F. Karsai, M. Schlipf, Vienna Ab-initio Simulation Package (VASP) 5.4.4 (2017), https://www.vasp.at.

[21] G. Kresse, J. Hafner, Ab initio Molecular-Dynamics Simulation of the Liquid-MetalAmorphous-Semiconductor Transition in Germanium, Phys. Rev. B 47 (1994) 14251-14269. https://doi.org/10.1103/PhysRevB.49.14251.

[22] G. Kresse, J. Furthmüller, Efficiency of Ab-Initio Total Energy Calculations for Metals and Semiconductors Using a Plane-Wave Basis Set, Comput. Mater. Sci. 6 (1996) 15-50. https://doi.org/10.1016/0927-0256(96)00008-0.

[23] G. Kresse, J. Furthmüller, Efficient Iterative Schemes for Ab Initio Total-Energy Calculations Using a Plane-Wave Basis Set, Phys. Rev. B 54 (1996) 11169-11186. https://doi.org/10.1103/PhysRevB.54.11169.

[24] J.P. Perdew, K. Burke, M. Ernzerhof, Generalized Gradient Approximation Made Simple, Phys. Rev. Lett. 77 (1996) 3865 3865-3868. https://doi.org/10.1103/PhysRevLett.77.3865.

[25] J.P. Perdew, K. Burke, M. Ernzerhof, Erratum: Generalized Gradient Approximation Made Simple, Phys. Rev. Lett. 78 (1997) 1396 1396. https://doi.org/10.1103/PhysRevLett.78.1396.

[26] H.J. Monkhorst, J.D. Pack, Special Points for Brillouin-Zone Integrations, Phys. Rev. B 13 (1976) 5188-5192. https://doi.org/10.1103/PhysRevB.13.5188.

[27] H.J. Monkhorst, J.D. Pack, "Special points for Brillouin-zone integrations" - A Reply, Phys. Rev. B 16 (1977) 1748-1749. https://doi.org/10.1103/PhysRevB.16.1748. 
[28] M. Methfessel, A.T. Paxton, High-Precision Sampling for Brillouin-Zone Integration in Metals, Phys. Rev. B 40 (1989) 3616-3621. https://doi.org/10.1103/PhysRevB.40.3616.

[29] G. Kresse, J. Hafner, Norm-Conserving and Ultrasoft Pseudopotentials for First-Row and Transition Elements, J. Phys.: Condens. Matter 6 (1994) 8245-8257. https://doi.org/10.1088/0953$\underline{8984 / 6 / 40 / 015}$.

[30] G. Kresse, D. Joubert, From Ultrasoft Pseudopotentials to the Projector Augmented-Wave Method, Phys. Rev. B 59 (1999) 1758-1775. https://doi.org/10.1103/PhysRevB.59.1758.

[31] J.D. Gale, General Utility Lattice Program (GULP) 5.1.1 (2019), http://gulp.curtin.edu.au/gulp/.

[32] J.D. Gale, Empirical Potential Derivation for Ionic Materials, Philos. Mag. B 73 (1996) 3-19. https://doi.org/10.1080/13642819608239107.

[33] J.D. Gale, GULP: A Computer Program for the Symmetry-adapted Simulation of Solids, J. Chem. Soc., Faraday Trans. 93 (1997) 629-637. https://doi.org/10.1039/A606455H.

[34] J.D. Gale, A.L. Rohl, The General Utility Lattice Program (GULP), Mol. Simul. 29 (2003) 291341. https://doi.org/10.1080/0892702031000104887.

[35] J.D. Gale, GULP: Capabilities and prospects, Z. Krist. 220 (2005) 552-554. https://doi.org/10.1524/zkri.220.5.552.65070.

[36] S. Plimpton, A. Thompson, S. Moore, A. Kohlmeyer, R. Berger, Large Atomic/Molecular Massively Parallel Simulator (LAMMPS) 17Nov2016 (2016), http://lammps.sandia.gov.

[37] S. Plimpton, Fast Parallel Algorithms for Short-Range Molecular Dynamics, J. Comput. Phys. 117 (1995) 1-19. https://doi.org/10.1006/jcph.1995.1039.

[38] A. Jewett, Moltemplate 1.34 (2015), https://www.moltemplate.org.

[39] A.I. Jewett, Z. Zhuang, J.-E. Shea, Moltemplate a Coarse-Grained Model Assembly Tool, Biophys. J. 104 (2013) 169a. https://doi.org/10.1016/j.bpj.2012.11.953.

[40] L. Verlet, Computer "Experiments" on Classical Fluids. I. Thermodynamical Properties of Lennard-Jones Molecules, Phys. Rev. A 159 (1967) 98-103. https://doi.org/10.1103/PhysRev.159.98. 
[41] S. Nosé, A Molecular Dynamics Method for Simulations in the Canonical Ensemble, Mol. Phys. 52 (1984) 255-268. https://doi.org/10.1080/00268978400101201.

[42] S. Nosé, A Unified Formulation of the Constant Temperature Molecular Dynamics Methods, J. Chem. Phys. 81 (1984) 511-519. https://doi.org/10.1063/1.447334.

[43] W.G. Hoover, Canonical Dynamics: Equilibrium Phase-space Distributions, Phys. Rev. A 1985 (1985) 1695-1697. https://doi.org/10.1103/PhysRevA.31.1695.

[44] W.G. Hoover, Constant-Pressure Equations of Motion, Phys. Rev. A 34 (1986) 2499-2500. https://doi.org/10.1103/PhysRevA.34.2499.

[45] R.W. Hockney, J.W. Eastwood, Computer Simulation Using Particles. 1st ed. Taylor \& Francis Group, Abingdon, UK, 1988. 